CERN/LAA/94-09

CERN-PPE/94-50

18 February 1994

\title{
INFLUENCE OF ELECTRON TRAPS ON CHARGE-COLLECTION EFFICIENCY IN GaAs RADIATION DETECTORS
}

\author{
F. NAVA*, C. CANALI \\ Facoltà di Ingegneria, Università di Modena, Italy \\ A. CASTALDINI, A. CAVALLINI \\ S. D'AURIA** , C. DEL PAPA \\ Dipartimento di Fisica dell'Università and \\ INFN, Bologna, Italy \\ C. FRIGERI, L. ZANOTTI \\ MASPEC/CNR, Parma, Italy \\ A. CETRONIO, C. LANZIERI \\ ALENIA SpA, Roma, Italy \\ A. ZICHICHI \\ CERN, Geneva, Switzerland
}

\begin{abstract}
This paper deals with the interpretation of the charge-collection efficiency for minimum ionizing particles in terms of electron traps in semi-insulating liquid encapsulated Czochralski gallium arsenide detectors fabricated with thicknesses of $80 \mu \mathrm{m}, 200 \mu \mathrm{m}$, and $300 \mu \mathrm{m}$. The results, obtained within the context of the RD-8 project, show that low values of chargecollection efficiency cannot be ascribed primarily to the presence of high concentrations of EL2 defects. Possible ways of increasing the charge-collection efficiency are briefly addressed.
\end{abstract}

(Submitted to Nuclear Instruments and Methods in Physics Research A)

\footnotetext{
* Also INFN, Bologna, Italy.

** Now at the University of Glasgow (UK).
} 



\section{INTRODUCTION}

Gallium arsenide Schottky detectors made of commercial undoped semi-insulating (SI) liquid encapsulated Czochralski (LEC) material have been shown to work as charged-particle detectors, with essentially $100 \%$ detection efficiency for minimum ionizing particles. Moreover the detectors have proved able to withstand radiation levels up to $50 \mathrm{Mrad}$ gamma rays and $7 \times 10^{14}$ neutrons per $\mathrm{cm}^{2}$, without severe degradation of their performance [1-4].

In spite of these very satisfactory properties, the charge-collection efficiency (cce) is found to be less than $100 \%$ for minimum ionizing particles, and this loss has been ascribed to the presence of deep levels in the materials which are responsible (i) for a depletion width $W$ of the detector smaller than expected [5] and (ii) for the trapping of a fraction of the charge carriers within this region [6].

In this work an attempt has been made to correlate the deep level densities with the charge collection using samples of LEC SI GaAs with different trap densities.

\section{EXPERIMENTAL PROCEDURE}

The detectors were made of undoped SI LEC <100> GaAs wafers supplied by Sumitomo and processed by ALENIA (which are hereafter called SL96) and those supplied by the MASPEC-CNR laboratory and processed at the Istituto Nazionale di Fisica Nucleare (INFN) Laboratory dedicated to R\&D of solid-state detectors at the Bologna section of the INFN (which are hereafter called LPA114/9 and LPA114/95). Resistivity and Hall mobility at room temperature were $1.5 \times 10^{7} \Omega \mathrm{cm}$ and $3900 \mathrm{~cm}^{2} / \mathrm{V}$ s for LPA114/9 specimens, $4 \times 10^{6}$ $\Omega \mathrm{cm}$ and $2700 \mathrm{~cm}^{2} / \mathrm{V} \mathrm{s}$ for LPA114/95 specimens, and $5.2 \times 10^{7} \Omega \mathrm{cm}$ and $6900 \mathrm{~cm}^{2} / \mathrm{V} \mathrm{s}$ for SL96 specimens. The Au-Ge-Ni metallization process was used as an alloyed ohmic contact for the SL96 specimen [7] whilst a non-alloyed ohmic contact from a solid-phase epitaxy growth of an $\mathrm{n}^{+} \mathrm{Si}$ doped layer on n-type GaAs was used for the specimens LPA114/9 and LPA114/95 [8]. For both specimens, the Schottky contact was realized by means of a circular dot of thin titanium film $(\sim 1000 \AA)$ electron-beam-deposited at a pressure of $(2-5) \times$ $10^{-7}$ Torr on the polished surface swabbed first with trichloroethylene and then ultrasonically cleaned with acetone and isopropyl alcohol, followed by a rinse in deionized water and in $\mathrm{HCl}: \mathrm{H}_{2} \mathrm{O}$ (1:1 by volume) until it was completely hydrophobic. A thin gold film, electronbeam-deposited in the same vacuum chamber on the titanium layer, formed the necessary electrical contact.

The cce was measured with the betas of a ${ }^{106} \mathrm{Ru}$ source as minimum ionizing particles, with the ohmic and the Schottky contacts of the GaAs detector grounded and set at a negative voltage, respectively. The signals, obtained at the Schottky contacts were amplified and shaped for a multichannel analyser [1,9] .

The thermal activation energy, the capture cross-sections, and the concentrations of the traps were determined by using photo-induced current transient spectroscopy (PICTS) [10]. The details of the measurements will be described elsewhere [11].

\section{RESULTS AND DISCUSSION}

In Fig. 1 the cce is shown as a function of reverse bias for the specimens examined. The main finding of this analysis is that the cce monotonically increases when the applied voltage is increased, but it always remains less than $100 \%$. The maximum value of the cce, measured at 
the breakdown voltage, is $34 \%, 56 \%$, and $75 \%$, for the LPA114/9, LPA114/95, and SL96 specimens, respectively. The reproducibility of the best value obtained for the cce has been verified by using several SL96 specimens. Moreover, since the cce is found to be strongly dependent on the thickness of the detector [4], the latter has been varied between 80 and $300 \mu \mathrm{m}$. Figure 2 shows the cce for $\beta$ on the Schottky contacts as a function of $V_{\mathrm{b}}$ and thickness for SL96 specimens. It is worth underlining that the maximum cce value $(\sim 80 \%)$ is well reproducible and almost independent of the thickness of the detector.

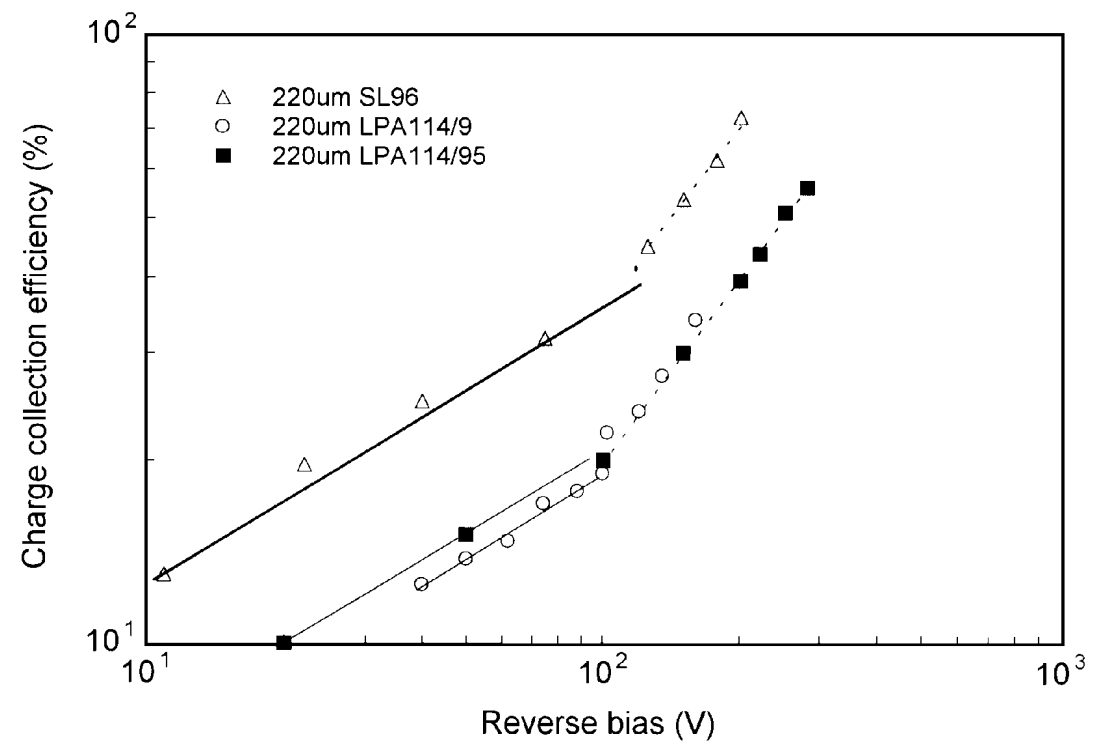

Fig. 1 Charge-collection efficiency for minimum ionizing particles as a function of reverse bias. The exponent $k$ in $V_{\mathrm{b}}^{k}$ dependence, measured by the slopes of the solid and dashed lines, takes the values 0.5 and 1 , respectively.

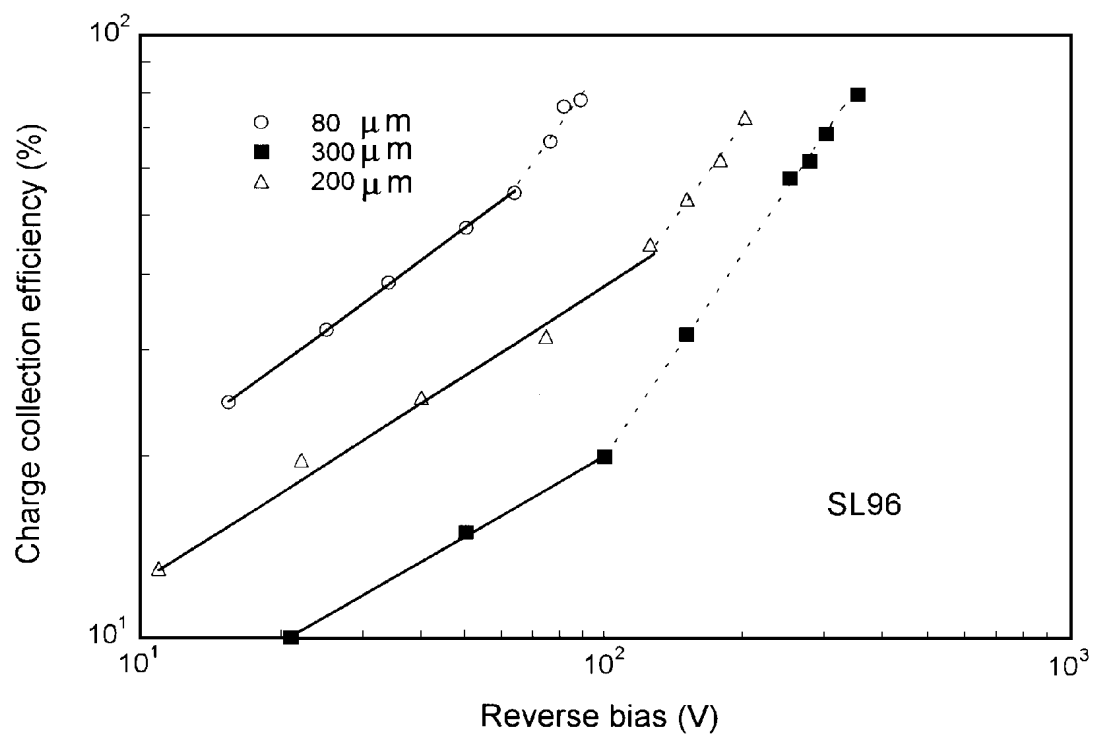


Fig. 2 Charge collection efficiency for minimum ionizing particles measured as a function of reverse bias with SL96 diodes of three different thicknesses. The solid and dashed lines have the same meaning as in Fig.1 .

Moreover the voltage dependence of the cce cannot be described by a unique power law of the type $V_{\mathrm{b}}^{k}$ over all the applied voltage range. Two regions can in fact be identified where the exponent $k$ is univocally determined by the values 0.5 and 1 respectively, and whose range measured in volts is a function of the diode thickness.

A possible explanation of these results can be found in the crystal purity of this material, which is not high. Semi-insulating LEC GaAs material is in fact compensated by a balance of native defect deep donors EL2 (located $0.8 \mathrm{eV}$ below the conduction band edge) and other donor impurities with shallow carbon acceptor impurities [12]. In thermal equilibrium the shallow impurities can be assumed to be fully ionized, while the deep donor levels are not fully ionized. They become ionized when a reverse bias is applied to the Schottky contact and start to trap most free charge carriers. This trapping, if slow compared with the amplifier drop, reduces the charge pulse height and hence the cce of the detector.

Moreover, their charge state and concentration alter the space-charge-region width and hence the electric-field distribution across the detector in a way which is not simple to interpret or predict by simulation of the detector's operation $[5,6,13]$.

In order to understand the characteristics of the material and the operating behaviour of the device so that the properties and performance can be optimized, it is essential to achieve an accurate knowledge of the traps present in the material in terms of activation energy and concentration. These parameters have been measured for the SL96, LPA114/9, and LPA114/95 specimens by means of the PICTS technique [11]. A typical spectrum is shown in Fig. 3a and the relative Arrhenius plots are shown in Figs. 3b, c, and d. The values of the activation energy for each trap, deduced from the slope of the straight lines, are indicated in the figures. Five types of traps can be identified. The first ranges from 0.75 to $0.82 \mathrm{eV}$ and corresponds to the EL2 defect; the second ranges from 0.53 to $0.58 \mathrm{eV}$; the third ranges from 0.39 to $0.41 \mathrm{eV}$; the fourth ranges from 0.32 to $0.34 \mathrm{eV}$; and the fifth ranges from 0.17 to $0.18 \mathrm{eV}$. The relative densities are reported in Fig. 4.

The correlation between trap concentration and cce for the three specimens is shown in Fig. 5.

The main features of this analysis are that i) the traps are electron traps and their energy levels are measured from the conduction band level, ii) the trap of $0.41 \mathrm{eV}$ is present in SL96 specimens at very low density $\left(<10^{13} \mathrm{~cm}^{-3}\right)$, iii) the density of EL2 $(0.75 \mathrm{eV})$ takes the lowest value in the SL96 specimens, and iv) the trap at $0.18 \mathrm{eV}$ is present at concentrations of the same order of magnitude as EL2 in SL96 specimens and almost one order of magnitude lower in LPA114/9 and LPA114/95 specimens.

These features seem to support the idea that the experimental limitation in the cce observed in these materials (Fig. 1) cannot only be ascribed to the presence of the deep level EL2, which is often assumed to play the leading role [6], but also to the defects in their totality. In general terms, in fact, all donors which lie in thermal equilibrium, near the Fermi energy level in the bulk of the detector, will be ionized when the band bending is increased by the application of the reverse bias to the Schottky barrier. They will add to the total positive charge leading to a deleterious perturbation of the electric field in the depletion region. 

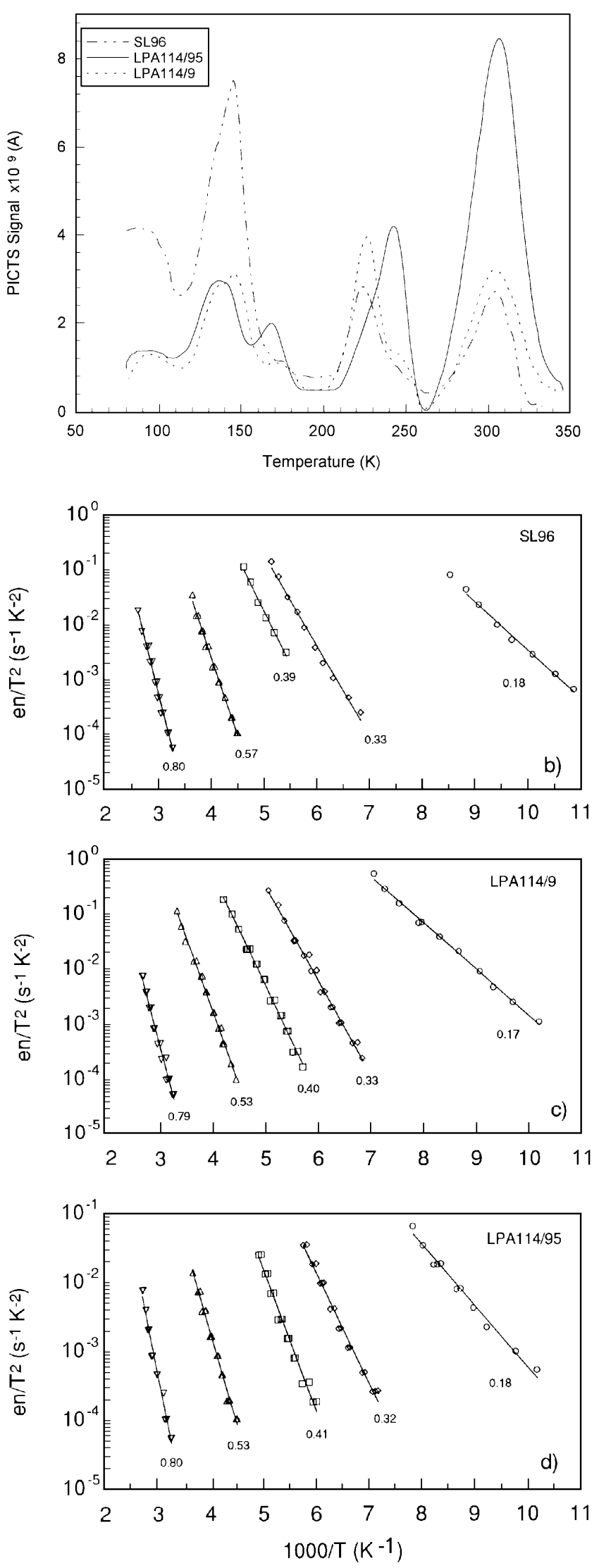

Fig. 3 a) PICTS spectra of SL96, LPA114/9, and LPA114/95 specimens obtained with an emission rate $e_{n}=10 \mathrm{~s}^{-1}$ and a filling pulse of $\left.30 \times 10^{-3} \mathrm{~s} . \mathrm{b}\right), \mathrm{c}$ ), and d) Arrhenius 
plots for the traps in the SL96, LPA114/9, and LPA114/95 specimens respectively. The activation energies of the traps are indicated alongside the relevant solid line.

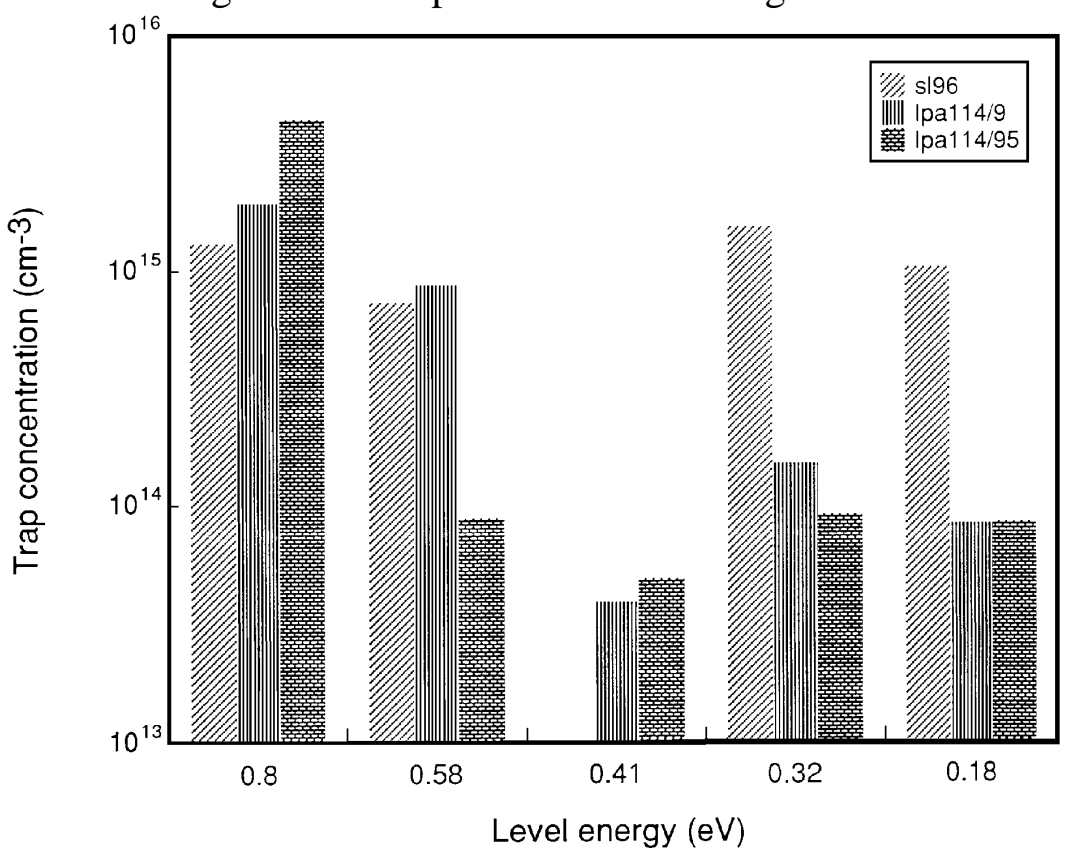

Fig. 4 Trap concentration found by PICTS data for the traps in Fig. 3.

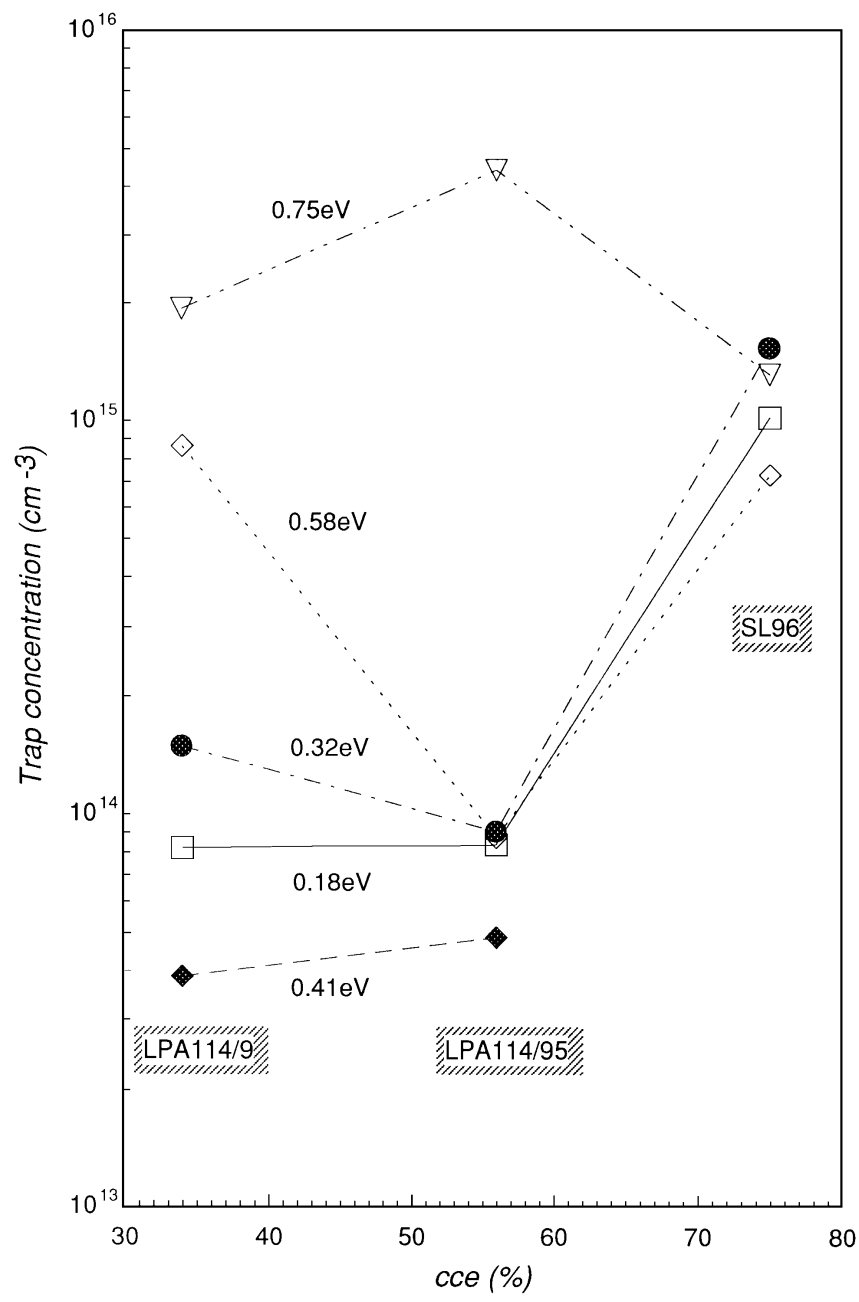


Fig. 5 Trap concentration as a function of the charge collection efficiency (cce) for minimum ionizing particles in the LPA114/9, LPA114/95, and SL96 diodes.

The shallow donor levels, however, can provide electrons to compensate the ionized deep level defects [14], so that these latter become neutral defects and cannot trap charge carriers and perturb the electric field.

Therefore, a possible explanation of the higher cce observed in SL96 specimens could be: i) the existence of two electronic states for the EL2 centre: EL2 ${ }^{+}$and EL2 ${ }^{0}$ and ii) the donorlike behaviour of the traps at $0.18 \mathrm{eV}$. The EL2+ is the ionized EL2 level which would exist in the starting material and would be generated after the application of a reverse bias to the Schottky contact. The neutral EL2 ${ }^{0}$ defect is the classical level which can emit its electron into the conduction band. The transition from the EL2 ${ }^{+}$to the neutral EL2 ${ }^{0}$ defect is assisted by the donors which provide the electrons for the compensation.

A similar compensation mechanism has been invoked to describe the quenching phenomena of photoconductance enhanced by proton irradiation in SI LEC GaAs [15].

\section{CONCLUSION}

Studies with minimum ionizing particles have shown that the cce is less than $100 \%$. Since it is not yet clear whether this is the result of short carrier lifetimes due to trapping or low carrier drift velocity due to a small electric field across the detector, in this work suitable techniques of analysis have been set up and detectors made with different materials have been examined in order to give an appropriate answer to the question. The results presented show that GaAs detectors have an acceptable performance in detecting minimum ionizing particles and there are good and reasonable indications to believe that the efficiency will approach $100 \%$ when the concentration of the traps is further decreased.

In addition, the comparison between the operation of particle detectors realized with bulk SI GaAs using different materials seems to indicate that the deleterious effect on the operation is to be expected from the ionized mid-gap electron traps, which are not perfectly compensated by shallow donor-like defects. The change observed in the exponent of the power law which describes the voltage dependence of the cce is surprising and not at present understood. Direct measurements of depletion width as a function of the applied voltage are in progress in order to understand this behaviour.

In conclusion, according to the indications of this preliminary investigation of LEC bulk GaAs, materials with a lower and equal concentration of deep donors and shallow dopant impurities will be utilized in future analyses to test the proposed hypothesis.

\section{Acknowledgements}

We wish to acknowledge the technical assistance we received from M. Curti and G. Mignoni (MASPEC-CNR). The contribution of C. Chiossi (Dip. Fisica Modena) was particularly appreciated. 


\section{References}

[1] R. Bertin et al., Nucl. Instrum. Methods A294 (1990) 211.

[2] S.P. Beaumont et al., Nucl. Instrum. Methods A321 (1992) 172.

[3] S.P. Beaumont et al., Nucl. Instrum. Methods A322 (1992) 472.

[4] S.P. Beaumont et al., Nucl. Instrum. Methods A 326 (1993) 313.

[5] RD8 Collaboration, Status Report CERN/DRDC (1993).

[6] D.S. McGregor et al., IEEE Trans. Nucl. Sci. 39 (1992) 1226.

[7] See, for example, N. Braslau, J.B. Gunn and J.L. Staples, Solid-State Electron. 10 (1967) 38.

[8] F. Nava, GaAs fabrication detector in Modena, in Proc. 20th INFN Eloisatron Workshop on GaAs Detectors and Electronics for High-Energy Physics, eds. K. Smith, C. del Papa and P.G. Pelfer (World Scientific, Singapore, 1992);

L.C. Wang, B. Zhang, F. Fang, E.D. Marshall, S.S. Lau, T. Sands and T.F. Kuech, J. Mater. Res. 3 (1988) 992.

[9] S.P. Beaumont et al., Proc. 3rd Int. Conf. on Advanced Technology and Particle Physics, Villa Olmo, Como, 1992. [Nucl. Phys. B32 (1993)].

[10] M. Tapiero , N. Benjelloun, J.P. Zielinger, S. El. Hamd, and C. Noguet, J. Appl. Phys. 64 (1988) 4006.

[11] A. Castaldini et al., Deep levels in LEC SI GaAs investigated by photo-induced current spectroscopy, submitted to J. Appl. Phys.

[12] R.K. Willardson and A. C. Beer, Semiconductors and semimetals (Academic Press, Orlando, USA, 1984), vol. 20.

[13] K. Berwick et al., Studies of Charge Collection in GaAs Radiation Detectors, to be published in Nucl. Instrum. Methods A.

[14] S.M. Sze, Physics of semiconductor devices (J. Wiley, New York, 1981), 2nd edn.

[15] K. Kuriyama, H. Takahashi, H. Kawahara, N. Hayashi, H. Watanabe and I. Sakamoto, J. Appl. Phys. 68 (1990) 6517. 